\title{
Analytical and experimental approach of the high density transverse flux linear stepper motor
}

\begin{abstract}
Detailed analytical and experimental approaches were performed to calculating the ratio of the pull-in thrust to the input power of High Density Transverse Flux linear Stepper Motor (HDTFLSM) with considerations of the electrical time constant, the mechanical time constant and the friction. The analytical approach involves the use of Fourier series analysis to derive the equation for the pull-in thrust to input power ratio. The experimentation was performed under different load conditions until the motor come to the standstill value. A chopped voltage was applied to motor at different speeds and the obtained results were compared with those for normal voltage. Comparison between analytical and experimental results shows good agreement, for instance at $0.22 \mathrm{~m} / \mathrm{s}$ the difference percentage on normal voltage and chopped voltage are $6.1 \%$ and $6.7 \%$, respectively. The chopped voltage has higher ratio of the pull-in thrust to the input power compare to normal voltage so that the machine performance can be improvised to a greater extent. The maximum improvement percentage of $18.8 \%$ on pull-in thrust to input power ratio for motor with chopped voltage was obtained at $0.22 \mathrm{~m} / \mathrm{s}$.
\end{abstract}

Keyword: High density; Linear motor; Pull-in thrust; Transverse flux; Stepper motor 